\title{
Impact of revised atlanta classification of acute pancreatitis on generation of a score employing modified CT severity index
}

Sir,

We read with great interest the article titled "Severity assessment of acute pancreatitis using CT severity index and modified CT severity index: Correlation with clinical outcomes and severity grading as per the Revised Atlanta Classification" by Sahu et al. published in the April-June 2017 issue of the Indian Journal of Radiology and Imaging. ${ }^{[1]}$ The manuscript is highly informative. We, however, would like to make the following pertinent observations.

One of the principal aims of the present study ${ }^{[1]}$ was to calculate the degree of concordance between the CT scoring indices and the clinical grading as per the Revised Atlanta Classification for Acute Pancreatitis. The former included CT Severity Index (CTSI) and Modified CT Severity Index (MCTSI), both of which generate a score based on CECT findings, thus, stratifying acute pancreatitis (AP) as mild, moderate, or severe. Revised Atlanta Classification for Acute Pancreatitis revolutionized the management of AP by simplifying clinical as well as morphological classifications and coining newer nomenclature for radiological findings to bring in more objectivity. ${ }^{[1,2]}$ Naturally, calculation of CT scoring indices, for e.g., MCTSI on the basis of newer terminology may alter the final score. For example:

a. Revised Atlanta Classification for Acute Pancreatitis considers any intrapancreatic collection straightaway parenchymal necrosis, ${ }^{[2]}$ which is in strong disagreement with the older terminology used to compute MCTSI. ${ }^{[3]}$ The latter grades intrapancreatic collection and parenchymal necrosis as two separate entities. ${ }^{[3]}$ Hence, MCTSI as per the latter (the older terminology) would be 2 or 4 (depending on the percentage of necrosis whether more than or less than $30 \%$ ), while the former would generate a score of 4

b. The nomenclature of fat islands in peripancreatic collections is yet another point of disagreement. ${ }^{[3]}$ While older terminology used to calculate MCTSI considers it as acute fluid collections without necrosis, ${ }^{[3]}$ revised terminology labels it to be acute necrotic collection (in acute stage). ${ }^{[2]}$

The present study defines the CT parameters as per the Revised Atlanta Classification for Acute Pancreatitis. ${ }^{[1]}$ However, MCTSI, if computed using the recent classification, may yield a different score compared to when calculated as per the older terminology. Hence, it would be of enormous help if the authors could clarify our doubts.

Financial support and sponsorship

Nil.

Conflicts of interest

There are no conflicts of interest. 


\section{Yashant Aswani, Prashant K Singh ${ }^{1}$}

Department of Radiology, Pacific Medical College and Hospital, Udaipur, 'Jhalawar Medical College, Rajasthan, India E-mail: aswaniyashant@gmail.com

\section{References}

1. Sahu B, Abbey P, Anand R, Kumar A, Tomer S, Malik E. Severity assessment ofacute pancreatitis using CT severity index and modified CT severity index: Correlation with clinical outcomes and severity grading as per the RevisedAtlanta Classification. Indian J Radiol Imaging 2017;27:152-60.

2. Thoeni RF. The revised Atlanta classification of acute pancreatitis: Its importance for the radiologist and its effect on treatment. Radiology 2012;262:751-64.

3. Sainani N, Catalano O, Sahani D. Pancreas. In: Haaga JR, Dogra VS, Forsting M, Gilkeson RC, Ha HK, Sundaram M, Editors. CT and MRI of the whole body. $5^{\text {th }}$ ed. Philadelphia: Mosby Elsevier; 2009. p. 1599-674.
This is an open access journal, and articles are distributed under the terms of the Creative Commons Attribution-NonCommercial-ShareAlike 4.0 License, which allows others to remix, tweak, and build upon the work non-commercially, as long as appropriate credit is given and the new creations are licensed under the identical terms.

\begin{tabular}{|l|l|}
\hline \multicolumn{2}{|c|}{ Access this article online } \\
\hline Quick Response Code: & Website: \\
& www.ijri.org \\
\hline & \\
& \\
\hline
\end{tabular}

Cite this article as: Aswani Y, Singh PK. Impact of revised atlanta classification of acute pancreatitis on generation of a score employing modified CT severity index. Indian J Radiol Imaging 2018;28:374-5.

() 2018 Indian Journal of Radiology and Imaging | Published by Wolters Kluwer - Medknow 